\title{
UNIFORM BOUNDS OF PILTZ DIVISOR PROBLEM OVER NUMBER FIELDS
}

\author{
WATARU TAKEDA
}

\begin{abstract}
We consider the upper bound of Piltz divisor problem over number fields. Piltz divisor problem is known as a generalization of the Dirichlet divisor problem. We deal with this problem over number fields and improve the error term of this function for many cases. Our proof uses the estimate of exponential sums. We also show uniform results for ideal counting function and relatively $r$-prime lattice points as one of applications.
\end{abstract}

\section{INTRODUCTION}

The behavior of arithmetic functions has long been studied and it is one of the most important research in analytic number theory. But many arithmetic functions $f(n)$ fluctuate as $n$ increases and it becomes difficult to deal with them. Thus many authors study partial sums $\sum_{n \leq x} f(n)$ to obtain some information about arithmetic functions $f(n)$. In this paper we consider Piltz divisor function $I_{K}^{m}(x)$ over number field. Let $K$ be a number field with extension degree $[K: \mathbf{Q}]=n$ and let $\mathcal{O}_{K}$ be its ring of integers. Let $D_{K}$ be absolute value of the discriminant of $K$. Then Piltz divisor function $I_{K}^{m}(x)$ counts the number of $m$-tuples of ideals $\left(\mathfrak{a}_{1}, \mathfrak{a}_{2}, \ldots, \mathfrak{a}_{m}\right)$ such that product of their ideal norm $\mathfrak{N a}_{1} \cdots \mathfrak{N a} \mathfrak{a}_{m} \leq x$. It is known that

$$
I_{K}^{m}(x) \sim \operatorname{Res}_{s=1}\left(\zeta_{K}(s)^{m} \frac{x^{s}}{s}\right) .
$$

We denote $\Delta_{K}^{m}(x)$ be the error term of $I_{K}^{m}(x)$, that is, $I_{K}^{m}(x)-\operatorname{Res}_{s=1}\left(\zeta_{K}(s)^{m} \frac{x^{s}}{s}\right)$.

The case of $m=1$ this function is the ordinary ideal counting function over $K$. For simplicity we substitute $I_{K}(x)$ and $\Delta_{K}(x)$ for $I_{K}^{1}(x)$ and $\Delta_{K}^{1}(x)$ respectively. There are many results about $I_{K}(x)$ from 1900's. In the case $K=\mathbf{Q}$, integer ideals of $\mathbf{Z}$ and positive integers are in one-to-one correspondence, so $I_{\mathbf{Q}}(x)=[x]$, where [.] is the Gauss symbol. For the general case, the best estimate of $\Delta_{K}(x)$ hitherto is the following theorem:

Theorem 1.2. The following estimates hold. For all $\varepsilon>0$

2010 Mathematics Subject Classification. 11N45 (primary),11R42, 11H06,11P21 (secondary). Key words and phrases. ideal counting function, exponential sum, Piltz divisor problem. 


\begin{tabular}{|c|c|c|}
\hline$n=[K: \mathbf{Q}]$ & $\Delta_{K}(x)$ & \\
\hline 2 & $O\left(x^{\frac{131}{116}}(\log x)^{\frac{18627}{8320}}\right.$ & Huxley. $\mathrm{Hu} 00$ \\
\hline 3 & $O\left(x^{\frac{43}{96}+\varepsilon}\right)$ & Müller. Mü88 \\
\hline 4 & $\frac{41}{22}+\varepsilon$ & Bordellès. Bo15. \\
\hline $5 \leq n \leq 10$ & $O\left(x^{1-\frac{4}{2 n+1}+\varepsilon}\right)$ & Bordellès. Bo15 \\
\hline $11 \leq n$ & $O\left(x^{1-\frac{3}{n+6}+\varepsilon}\right)$ & Lao. La10] \\
\hline
\end{tabular}

There are also many results about $I_{\mathbf{Q}}^{m}$ from 1800's. In 1849 Dirichlet shows that

$$
I_{\mathbf{Q}}^{2}(x)=x \log x+(2 \gamma-1) x+O\left(x^{\frac{1}{2}}\right),
$$

where $\gamma$ is the Euler constant, defined by the equation

$$
\gamma=\lim _{n \rightarrow \infty}\left(\sum_{k=1}^{n} \frac{1}{k}-\log n\right) .
$$

The $O$-term is improved by many researchers many times, the best estimate hitherto is $x^{\frac{517}{1648}+\varepsilon}$ BW17.

As we have mentioned above, there exists many results about other divisor problems but it seems that there are not many results about piltz divisor problem over number fields. In 1993, Nowak shows the following theorem:

Theorem 1.3 (Nowak [No93]). When $n=[K: \mathbf{Q}] \geq 2$, then we get

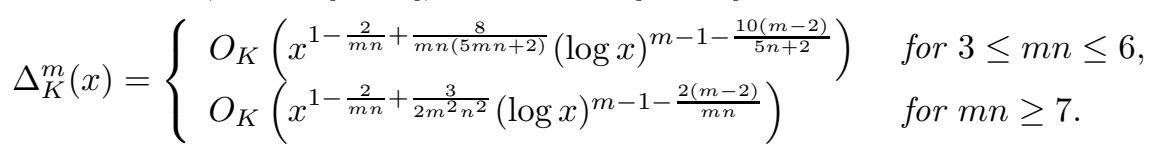

For the estimate of lower bound, Girstmair, Kühleitner, Müller and Nowak obtain the following $\Omega$-results:

Theorem 1.4 (Girstmair, Kühleitner, Müller and Nowak GKMN05]). For any fixed number field $K$ with $n=[K: \mathbf{Q}] \geq 2$

$$
\Delta_{K}^{m}(x)=\Omega\left(x^{\frac{1}{2}-\frac{1}{2 m n}}(\log x)^{\frac{1}{2}-\frac{1}{2 m n}}(\log \log x)^{\kappa}(\log \log \log x)^{-\lambda}\right),
$$

where $\kappa$ and $\lambda$ are constants depending on $K$. To be more precise, let $K^{\text {gal }}$ be the Galois closure of $K / \mathbf{Q}, G=\operatorname{Gal}\left(K^{\text {gal }} / \mathbf{Q}\right)$ its Galois group and $H=G a l\left(K^{\text {gal }} / K\right)$ the subgroup of $G$ corresponding to $K$. Then

$$
\kappa=\frac{m n+1}{2 m n}\left(\sum_{\nu=1}^{n} \delta_{\nu} \nu^{\frac{2 m n}{m n+1}}-1\right) \text { and } \lambda=\frac{m n+1}{4 m n} R+\frac{m n-1}{2 m n},
$$

where

$$
\delta_{\nu}=\frac{\left|\left\{\tau \in G||\left\{\sigma \in G \mid \tau \in \sigma H \sigma^{-1}\right\}|=\nu| H \mid\right\}\right|}{|G|}
$$

and $R$ is the number of $1 \leq \nu \leq n$ with $\delta_{\nu}>0$.

We know the following conditional result:

If we assume the Lindelöf hypothsis for Dedekind zeta function, it holds that for all $\varepsilon>0$, for all $K$ and for all $m$

$$
\Delta_{K}^{m}(x)=O_{\varepsilon}\left(x^{\frac{1}{2}+\varepsilon} D_{K}^{\varepsilon}\right) .
$$


In this paper we estimate the error term of $\Delta_{K}^{m}(x)$ by using exponential sums. In No93 and GKMN05, they use other approaches, so we expect new development for the Piltz divisor problem over number field. As a results, we improve the estimate of upper bound of $\Delta_{K}^{m}(x)$ for many $K$ and many $m$.

In Section 2, we show some auxiliary theorems to consider the upper bound of the error term $\Delta_{K}^{m}(x)$. First we give a review of the convexity bound for the Dedekind zeta function and generalized Atkinson's Lemma At41. Next we show proposition 2.6] which reduces an ideal counting problem to an exponential sums problem. This proposition plays a crucial role in our computing $\Delta_{K}^{m}(x)$.

In Section 3, we prove the following theorem about the error term $\Delta_{K}^{m}(x)$ by using estimate of exponential sums.

Theorem 1.7. For every $\varepsilon>0$ the following estimates hold. When $m n \geq 4$, then

$$
\Delta_{K}^{m}(x)=O_{n, m, \varepsilon}\left(x^{\frac{2 m n-3}{2 m n+1}+\varepsilon} D_{K}^{\frac{2 m}{2 m n+1}+\varepsilon}\right) .
$$

This theorem gives improvement of upper bound of $\Delta_{K}^{m}(x)$ for $m n \geq 4$.

In Section 4, we give some application. First we give an uniform estimate for ideal counting function over number fields. Second we show a good uniform upper bound of the distribution of relatively $r$-prime lattice points over number fields as a corollary of the first application.

In Section 5, we consider a conjecture about estimates for Piltz divisor functions over number field. It is proposed that for all number fields $K$ and for all $m$ the best upper bound of the error term is better than that on the assumption of the Lindelöf Hypothesis (1.6). If $m n \leq 3$ this conjecture holds, but the other cases it seems to be very difficult.

\section{Auxiliary Theorem}

In this section, we show some important lemmas for our argument. Let $s=\sigma+i t$ and $n=[K: \mathbf{Q}]$. We use the convexity bound of Dedekind zeta function to obtain an upper bound of the error term of Piltz divisor function $\Delta_{K}^{m}(x)$.

It is well-known fact that Dedekind zeta function satisfies the following functional equation:

$$
\zeta_{K}(1-s)=D_{K}^{s-\frac{1}{2}} 2^{n(1-s)} \pi^{-n s} \Gamma(s)^{n}\left(\cos \frac{\pi s}{2}\right)^{r_{1}+r_{2}}\left(\sin \frac{\pi s}{2}\right)^{r_{2}} \zeta_{K}(s),
$$

where $r_{1}$ is the number of real embeddings of $K$ and $r_{2}$ is the number of pairs of complex embeddings,

The Phragmen-Lindelöf principle and (2.1) give the well-known convexity bound of the Dedekind zeta function [Ra59]: For any $\varepsilon>0$ and $n=[K: \mathbf{Q}]$

$$
\zeta_{K}(\sigma+i t)= \begin{cases}O_{n, \varepsilon}\left(|t|^{\frac{n}{2}-n \sigma+\varepsilon} D_{K}^{\frac{1}{2}-\sigma+\varepsilon}\right) & \text { if } \sigma \leq 0, \\ O_{n, \varepsilon}\left(|t|^{\frac{n(1-\sigma)}{2}+\varepsilon} D_{K}^{\frac{1-\sigma}{2}+\varepsilon}\right) & \text { if } 0 \leq \sigma \leq 1, \\ O_{n, \varepsilon}\left(|t|^{\varepsilon} D_{K}^{\varepsilon}\right) & \text { if } 1 \leq \sigma\end{cases}
$$

as $|t|^{n} D_{K} \rightarrow \infty$, where $K$ runs through number fields with $[K: \mathbf{Q}]=n$. In the previous papers, we also use this convexity bound (2.2) to estimate the distribution of ideals. In the following sections, we show some estimate for $\Delta_{K}^{m}(x)$ in the similar way to our previous papers. 
Lemma 2.3 states the growth of the product of Gamma function and trigonometric functions in the functional equation (2.1) of Dedekind zeta function.

Lemma 2.3. Let $\tau \in\{\cos , \sin \}$ and $n$ be a positive integer

$$
\begin{aligned}
& \frac{\Gamma(s)^{n}}{1-s}\left(\cos \frac{\pi s}{2}\right)^{r_{1}+r_{2}}\left(\sin \frac{\pi s}{2}\right)^{r_{2}} \\
= & C n^{-n s} \Gamma\left(n s-\frac{n+1}{2}\right) \tau\left(\frac{n \pi s}{2}\right)+O_{n}\left(|t|^{-2+n \sigma-\frac{n}{2}}\right),
\end{aligned}
$$

where $C$ is a constant and $s=\sigma+i t$.

Proof. This lemma is shown from the Stirling formula and estimate for trigonometric function.

Next we introduce the generalized Atkinson's lemma. This lemma is quite useful for calculating integrals of the Dedekind zeta function.

Lemma 2.4 (Atkinson At41]). Let $y>0,1<A \leq B$ and $\tau \in\{\cos , \sin \}$, and we define

$$
I=\frac{1}{2 \pi i} \int_{A-i B}^{A+i B} \Gamma(s) \tau\left(\frac{\pi s}{2}\right) y^{-s} d s
$$

If $y \leq B$, then

$$
I=\tau(y)+O\left(y^{-\frac{1}{2}} \min \left(\left(\log \frac{B}{y}\right)^{-1}, B^{\frac{1}{2}}\right)+y^{-A} B^{A-\frac{1}{2}}+y^{-\frac{1}{2}}\right) .
$$

If $y>B$, then

$$
I=O\left(y^{-A}\left(B^{A-\frac{1}{2}} \min \left(\left(\log \frac{y}{B}\right)^{-1}, B^{\frac{1}{2}}\right)+A^{A-\frac{1}{2}}\right)\right) .
$$

Finally we introduce the following lemma to reduce the ideal counting problem to an exponential sum problem.

Lemma 2.5 (Bordellès [Bo15]). Let $1 \leq L \leq R$ be a real number and $f$ be an arithmetical function satisfying $f(m)=O\left(m^{\varepsilon}\right)$, and let $\boldsymbol{e}(x)=\exp (2 \pi i x)$ and $F=f * \mu$, where $*$ is the Dirichlet product symbol. For $a \in \mathbf{R}-\{1\}, b, x \in \mathbf{R}$ and for every $\varepsilon>0$ the following estimate holds.

$$
\begin{aligned}
& \sum_{m \leq R} \frac{f(m)}{m^{a}} \tau\left(2 \pi x m^{b}\right)
\end{aligned}
$$

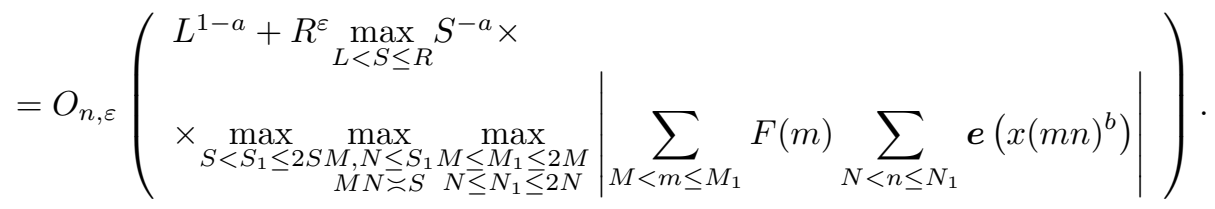

Next proposition plays a crucial role in our computing $I_{K}^{m}(x)$. We consider the distribution of ideals of $\mathcal{O}_{K}$, where $K$ runs through extensions with $[K: \mathbf{Q}]=n$ and some conditions. The detail of the conditions will be determined later, but they state the relation of the principal term and the error term. 
Proposition 2.6. Let $F_{K}=I_{K}^{m} * \mu$. For every $\varepsilon>0$ the following estimate holds.

$$
\begin{aligned}
& \Delta_{K}^{m}(x) \\
& =O_{n, m, \varepsilon}\left(\begin{array}{l}
L^{1-\alpha}+x^{\frac{m n-1}{2 m n}} D_{K}^{\frac{1}{2 n}} R^{\varepsilon} \max _{L \leq S \leq R} S^{-\frac{m n+1}{2 m n}} \times \\
\times \max _{\substack{S<S_{1} \leq 2 S S M, N \leq S_{1} M \leq M_{1} \leq 2 M \\
M N \cong S}} \max _{\substack{N \leq N_{1} \leq 2 N \\
M<l \leq M_{1}}} F_{K}(m) \sum_{\substack{N<k \leq N_{1} \\
\text { and }}} \boldsymbol{e}\left(m n\left(\frac{x l k}{D_{K}}\right)^{\frac{1}{m n}}\right) \mid \\
+x^{\frac{m n-2}{2 m n}+\varepsilon} D_{K}^{\frac{1}{n}+\varepsilon} R^{\frac{m n-2}{2 m n}+\varepsilon}+x^{\frac{m n-1}{m n}+\varepsilon} D_{K}^{\frac{1}{n}+\varepsilon} R^{-\frac{1}{m n}+\varepsilon}
\end{array}\right) .
\end{aligned}
$$

where $K$ runs through number fields with $[K: \mathbf{Q}]=n$ and some conditions.

Proof. Let $d_{K}^{m}(l)$ be the number of $m$-tuples of ideals $\left(\mathfrak{a}_{1}, \mathfrak{a}_{2}, \ldots, \mathfrak{a}_{m}\right)$ such that product of their ideal norm $\mathfrak{N a}_{1} \cdots \mathfrak{N a} \mathfrak{a}_{m}=l$. Then one can check it easily that

$$
\zeta_{K}(s)^{m}=\sum_{l=1}^{\infty} \frac{d_{K}^{m}(l)}{l^{s}} \text { for } \Re s>1
$$

and

$$
I_{K}^{m}(x)=\sum_{l \leq x} d_{K}^{m}(l)
$$

Thus Perron's formula plays a crucial role in this proof.

We consider the integral

$$
\frac{1}{2 \pi i} \int_{C} \zeta_{K}(s)^{m} \frac{x^{s}}{s} d s
$$

where $C$ is the contour $C_{1} \cup C_{2} \cup C_{3} \cup C_{4}$ shown in the following Figure 1,

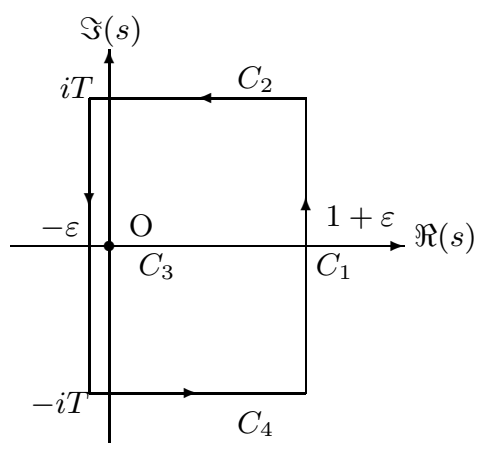

FiguRE 1.

In a way similar to the well-known proof of Perron's formula, we estimate

$$
\frac{1}{2 \pi i} \int_{C_{1}} \zeta_{K}(s)^{m} \frac{x^{s}}{s} d s=I_{K}^{m}(x)+O_{\varepsilon}\left(\frac{x^{1+\varepsilon}}{T}\right) .
$$

We can select the large $T$, so that the $O$-term in the right hand side is sufficiently small. For estimating the left hand side by using estimate (2.2), we divide it into the integrals over $C_{2}, C_{3}$ and $C_{4}$. 
First we consider the integrals over $C_{2}$ and $C_{4}$ as

$$
\begin{aligned}
& \left|\frac{1}{2 \pi i} \int_{C_{2} \cup C_{4}} \zeta_{K}(s)^{m} \frac{x^{s}}{s} d s\right| \\
\leq & \frac{1}{2 \pi} \int_{-\varepsilon}^{1+\varepsilon}\left|\zeta_{K}(\sigma+i T)\right|^{m} \frac{x^{\sigma}}{T} d \sigma+\frac{1}{2 \pi} \int_{-\varepsilon}^{1+\varepsilon}\left|\zeta_{K}(\sigma-i T)\right|^{m} \frac{x^{\sigma}}{T} d \sigma .
\end{aligned}
$$

It holds by the convexity bound of Dedekind zeta function (2.2) that their sum is estimated as

$$
\begin{aligned}
\left|\frac{1}{2 \pi i} \int_{C_{2} \cup C_{4}} \zeta_{K}(s)^{m} \frac{x^{s}}{s} d s\right| & =O_{n, m, \varepsilon}\left(\int_{-\varepsilon}^{1+\varepsilon}\left(T^{m n} D_{K}^{m}\right)^{\frac{1-\sigma}{2}+\varepsilon} \frac{x^{\sigma}}{T} d \sigma\right) \\
& =O_{n, m, \varepsilon}\left(\frac{x^{1+\varepsilon} D_{K}^{\varepsilon}}{T^{1-\varepsilon}}+T^{\frac{m n}{2}-1+\varepsilon} D_{K}^{\frac{m}{2}}+\varepsilon x^{-\varepsilon}\right) .
\end{aligned}
$$

By the Cauchy residue theorem, (2.8) and (2.9) we obtain

$$
\Delta_{K}^{m}(x)=\int_{C_{3}} \zeta_{K}(s)^{m} \frac{x^{s}}{s} d s+O_{n, m, \varepsilon}\left(\frac{x^{1+\varepsilon} D_{K}^{\varepsilon}}{T^{1-\varepsilon}}+T^{\frac{m n}{2}-1+\varepsilon} D_{K}^{\frac{m}{2}+\varepsilon} x^{-\varepsilon}\right) .
$$

Thus it suffices to consider the integral over $C_{3}$ as

$$
\frac{1}{2 \pi i} \int_{C_{3}} \zeta_{K}(s)^{m} \frac{x^{s}}{s} d s=\frac{1}{2 \pi i} \int_{-\varepsilon-i T}^{-\varepsilon+i T} \zeta_{K}(s)^{m} \frac{x^{s}}{s} d s .
$$

Changing the variable $s$ to $1-s$, we have

$$
\frac{1}{2 \pi i} \int_{C_{3}} \zeta_{K}(s)^{m} \frac{x^{s}}{s} d s=\frac{1}{2 \pi i} \int_{1+\varepsilon-i T}^{1+\varepsilon+i T} \zeta_{K}(1-s)^{m} \frac{x^{1-s}}{1-s} d s
$$

From this functional equation (2.1), it holds that

$$
\begin{aligned}
& \frac{1}{2 \pi i} \int_{C_{3}} \zeta_{K}(s)^{m} \frac{x^{s}}{s} d s \\
= & \frac{1}{2 \pi i} \int_{1+\varepsilon-i T}^{1+\varepsilon+i T}\left(D_{K}^{s-\frac{1}{2}} 2^{n(1-s)} \pi^{-n s} \Gamma(s)^{n}\left(\cos \frac{\pi s}{2}\right)^{r_{1}+r_{2}}\left(\sin \frac{\pi s}{2}\right)^{r_{2}} \zeta_{K}(s)\right)^{m} \frac{x^{1-s}}{1-s} d s .
\end{aligned}
$$

By lemma 2.3 the integral over $C_{3}$ can be expressed as

$$
\begin{aligned}
& \frac{1}{2 \pi i} \int_{C_{3}} \zeta_{K}(s) \frac{x^{s}}{s} d s \\
= & \frac{C x}{2 \pi i} \int_{1+\varepsilon-i T}^{1+\varepsilon+i T} D_{K}^{-\frac{m}{2}}\left(\frac{(2 n)^{m n} \pi^{m n} x}{D_{K}^{m}}\right)^{-s} \Gamma\left(m n s-\frac{m n+1}{2}\right) \tau\left(\frac{m n \pi s}{2}\right) \zeta_{K}(s) d s \\
& +O_{n, m, \varepsilon}\left(D_{K}^{\frac{m}{2}+\varepsilon} T^{\frac{m n}{2}-1+\varepsilon} x^{-\varepsilon}\right) .
\end{aligned}
$$


Changing the variable $m n s-\frac{m n+1}{2}$ to $s$, we have

$$
\begin{aligned}
& \frac{1}{2 \pi i} \int_{C_{3}} \zeta_{K}(s) \frac{x^{s}}{s} d s \\
= & \frac{C x^{\frac{m n-1}{2 m n}} D_{K}^{\frac{1}{2 n}}}{2 \pi i} \int_{\frac{m n-1}{2}+m n \varepsilon-m n i T}^{\frac{m n-1}{2}+m n \varepsilon+m n i T}\left(2 m n \pi\left(\frac{x}{D_{K}^{m}}\right)^{\frac{1}{m n}}\right)^{-s} \Gamma(s) \tau\left(\frac{\pi s}{2}+\frac{(m n+1) \pi}{4}\right) \\
& \times \zeta_{K}\left(\frac{s}{m n}+\frac{m n+1}{2 m n}\right) d s+O_{n, m, \varepsilon}\left(D_{K}^{\frac{m}{2}+\varepsilon} T^{\frac{m n}{2}-1+\varepsilon} x^{-\varepsilon}\right) .
\end{aligned}
$$

From (2.7) the function $\zeta_{K}(s)^{m}$ can be expressed as a Dirichlet series. It is absolutely and uniformly convergent on compact subsets on $\Re(s)>1$. Therefore we can interchange the order of summation and integral. Thus we obtain

$$
\begin{aligned}
& \int\left(2 m n \pi\left(\frac{x}{D_{K}^{m}}\right)^{\frac{1}{m n}}\right)^{-s} \Gamma(s) \tau\left(\frac{\pi s}{2}+\frac{(m n+1) \pi}{4}\right) \zeta_{K}\left(\frac{s}{m n}+\frac{m n+1}{2 m n}\right) d s \\
= & \sum_{l=1}^{\infty} \frac{d_{K}^{m}(l)}{l^{\frac{m n+1}{2 m n}}} \int\left(2 m n \pi\left(\frac{l x}{D_{K}^{m}}\right)^{\frac{1}{m n}}\right)^{-s} \Gamma(s) \tau\left(\frac{\pi s}{2}+\frac{(m n+1) \pi}{4}\right) d s,
\end{aligned}
$$

where the integration is on the vertical line from $\frac{m n-1}{2}+m n \varepsilon-m n i T$ to $\frac{m n-1}{2}+$ $m n \varepsilon+m n i T$. Properties of trigonometric function lead to

$$
\tau\left(\frac{\pi s}{2}+\frac{(m n+1) \pi}{4}\right)= \pm \begin{cases}\tau\left(\frac{\pi s}{2}\right) & \text { if } m n \text { is odd } \\ \frac{1}{\sqrt{2}}\left(\tau\left(\frac{\pi s}{2}\right) \pm \tau_{1}\left(\frac{\pi s}{2}\right)\right) & \text { if } m n \text { is even }\end{cases}
$$

where $\left\{\tau, \tau_{1}\right\}=\{\sin , \cos \}$. Hence it holds that

$$
\begin{aligned}
& \frac{1}{2 \pi i} \int_{C_{3}} \zeta_{K}(s)^{m} \frac{x^{s}}{s} d s \\
= & \frac{C x^{\frac{m n-1}{2 m n}} D_{K}^{\frac{1}{2 n}}}{2 \pi i} \sum_{l=1}^{\infty} \frac{d_{K}^{m}(l)}{l^{\frac{m n+1}{2 m n}}} \int_{\frac{m n-1}{2}+m n \varepsilon-m n i T}^{\frac{m n-1}{2}+m n \varepsilon+m n i T}\left(2 m n \pi\left(\frac{l x}{D_{K}^{m}}\right)^{\frac{1}{m n}}\right)^{-s} \Gamma(s) \tau\left(\frac{\pi s}{2}\right) d s \\
& +O_{n, m, \varepsilon}\left(D_{K}^{\frac{m}{2}+\varepsilon} T^{\frac{m n}{2}-1+\varepsilon} x^{-\varepsilon}\right) .
\end{aligned}
$$


Now we apply lemma 2.4 to this integral with $y=2 m n \pi\left(\frac{l x}{D_{K}^{m}}\right)^{\frac{1}{m n}}, A=\frac{m n-1}{2}+$ $m n \varepsilon, B=m n T$ and $T=2 \pi\left(\frac{x R}{D_{K}^{m}}\right)^{\frac{1}{m n}}$, this becomes

$$
\begin{aligned}
& \frac{1}{2 \pi i} \int_{C_{3}} \zeta_{K}(s)^{m} \frac{x^{s}}{s} d s \\
= & \frac{C x^{\frac{m n-1}{2 m n}} D_{K}^{\frac{1}{2 n}}}{2 \pi i} \sum_{l \leq R} \frac{d_{K}^{m}(l)}{l^{\frac{m n+1}{2 m n}}} \tau\left(2 m n \pi\left(\frac{l x}{D_{K}^{m}}\right)^{\frac{1}{m n}}\right) \\
& +O_{n, m, \varepsilon}\left(x^{\frac{m n-2}{2 m n}} D_{K}^{\frac{1}{n}} \sum_{l \leq R} \frac{d_{K}^{m}(l)}{l^{\frac{m n+2}{2 m n}}} \min \left\{\left(\log \frac{R}{l}\right)^{-1},\left(\frac{R x}{D_{K}^{m}}\right)^{\frac{1}{2 m n}}\right\}\right) \\
& +O_{n, m, \varepsilon}\left(x^{\frac{m n-2}{2 m n}} D_{K}^{\frac{1}{n}} \sum_{l \leq R} \frac{d_{K}^{m}(l)}{l^{\frac{m n+2}{2 m n}}}\left(\left(\frac{R}{l}\right)^{\frac{m n-2}{2 m n}}+1\right)\right) \\
& +O_{n, m, \varepsilon}\left(x^{\frac{m n-2}{2 m n}} D_{K}^{\frac{1}{n}} R^{\frac{m n-2}{2 m n}+\varepsilon} \sum_{l>R} \frac{d_{K}^{m}(l)}{l^{1+\varepsilon}} \min \left\{\left(\log \frac{l}{R}\right)^{-1},\left(\frac{R x}{D_{K}^{m}}\right)^{\frac{1}{2 m n}}\right\}\right) \\
& +O_{n, m, \varepsilon}\left(x^{\frac{m n-2}{2 m n}+\varepsilon} D_{K}^{\frac{1}{n}+\varepsilon} R^{\frac{m n-2}{2 m n}+\varepsilon}\right) .
\end{aligned}
$$

We evaluate three $O$-terms as follows.

First we consider the first $O$-term. One can estimate $\left(\log \frac{R}{l}\right)^{-1}=O\left(\frac{R}{R-l}\right)$, so we obtain

$$
\begin{aligned}
& O_{n, m, \varepsilon}\left(x^{\frac{m n-2}{2 m n}} D_{K}^{\frac{1}{n}} \sum_{l \leq R} \frac{d_{K}^{m}(l)}{l^{\frac{m n+2}{2 m n}}} \min \left\{\left(\log \frac{R}{l}\right)^{-1},\left(\frac{R x}{D_{K}^{m}}\right)^{\frac{1}{2 m n}}\right\}\right) \\
= & O_{n, m, \varepsilon}\left(x^{\frac{m n-2}{2 m n}} D_{K}^{\frac{1}{n}} \sum_{l \leq[R]-1} \frac{d_{K}^{m}(l)}{l^{\frac{m n+2}{2 m n}}}\left(\log \frac{R}{l}\right)^{-1}+x^{\frac{m n-2}{2 m n}} D_{K}^{\frac{1}{n}} \sum_{[R] \leq l \leq R} \frac{d_{K}^{m}(l)}{l^{\frac{m n+2}{2 m n}}}\left(\frac{R x}{D_{K}^{m}}\right)^{\frac{1}{2 m n}}\right) \\
= & O_{n, m, \varepsilon}\left(x^{\frac{m n-2}{2 m n}} D_{K}^{\frac{1}{n}} \sum_{l \leq[R]-1} \frac{d_{K}^{m}(l)}{l^{\frac{m n+2}{2 m n}}} \frac{R}{R-l}+x^{\frac{m n-1}{2 m n}} D_{K}^{\frac{1}{2 n}} R^{\frac{1}{2 m n}} \sum_{[R] \leq l \leq R} \frac{d_{K}^{m}(l)}{l^{\frac{m n+2}{2 m n}}}\right) \\
= & O_{n, m, \varepsilon}\left(x^{\frac{m n-2}{2 m n}} D_{K}^{\frac{1}{n}} R^{\frac{m n-2}{2 m n}+\varepsilon}+x^{\frac{m n-1}{2 m n}} D_{K}^{\frac{1}{2 n}} R^{-\frac{m n+1}{2 m n}}\right) .
\end{aligned}
$$

Next we calculate the second $O$-term.

$$
O_{n, m, \varepsilon}\left(x^{\frac{m n-2}{2 m n}} D_{K}^{\frac{1}{n}} \sum_{l \leq R} \frac{d_{K}^{m}(l)}{l^{\frac{m n+2}{2 m n}}}\left(\left(\frac{R}{l}\right)^{\frac{m n-2}{2 m n}}+1\right)\right)=O_{n, m, \varepsilon}\left(x^{\frac{m n-2}{2 m n}} D_{K}^{\frac{1}{n}} R^{\frac{m n-2}{2 m n}} \sum_{l \leq R} \frac{d_{K}^{m}(l)}{l}\right) .
$$


Since it is well-known that $d_{K}^{m}(l)=O\left(l^{\varepsilon}\right)$, we get

$$
\begin{aligned}
O_{n, m, \varepsilon}\left(x^{\frac{m n-2}{2 m n}} D_{K}^{\frac{1}{n}} \sum_{l \leq R} \frac{d_{K}^{m}(l)}{l^{\frac{m n+2}{2 m n}}}\left(\left(\frac{R}{l}\right)^{\frac{m n-2}{2 m n}}+1\right)\right) & =O_{n, m, \varepsilon}\left(x^{\frac{m n-2}{2 m n}} D_{K}^{\frac{1}{n}} R^{\frac{m n-2}{2 m n}} \int_{1}^{R} \frac{t^{\varepsilon}}{t} d t\right) \\
& =O_{n, m, \varepsilon}\left(x^{\frac{m n-2}{2 m n}} D_{K}^{\frac{1}{n}} R^{\frac{m n-2}{2 m n}+\varepsilon}\right) .
\end{aligned}
$$

Finally we estimate the third $O$-term in a similar way to calculate the first $O$-term. One can estimate $\left(\log \frac{l}{R}\right)^{-1}=O\left(\frac{R}{l-R}\right)$, so we obtain

$$
\begin{aligned}
& O_{n, m, \varepsilon}\left(x^{\frac{m n-2}{2 m n}} D_{K}^{\frac{1}{n}} R^{\frac{m n-2}{2 m n}+\varepsilon} \sum_{l>R} \frac{d_{K}^{m}(l)}{l^{1+\varepsilon}} \min \left\{\left(\log \frac{l}{R}\right)^{-1},\left(\frac{R x}{D_{K}^{m}}\right)^{\frac{1}{2 m n}}\right\}\right) \\
= & O_{n, m, \varepsilon}\left(x^{\frac{m n-2}{2 m n}} D_{K}^{\frac{1}{n}} R^{\frac{m n-2}{2 m n}+\varepsilon}\left(\sum_{R<l \leq[R]+1} \frac{d_{K}^{m}(l)}{l^{1+\varepsilon}}\left(\frac{R x}{D_{K}^{m}}\right)^{\frac{1}{2 m n}}+\sum_{[R]+2 \leq l} \frac{d_{K}^{m}(l)}{l^{1+\varepsilon}}\left(\log \frac{l}{R}\right)^{-1}\right)\right) \\
= & O_{n, m, \varepsilon}\left(x^{\frac{m n-1}{2 m n}} D_{K}^{\frac{1}{2 n}} R^{\frac{m n-1}{2 m n}+\varepsilon} \sum_{R<l \leq[R]+1} \frac{d_{K}^{m}(l)}{l^{1+\varepsilon}}+x^{\frac{m n-2}{2 m n}} D_{K}^{\frac{1}{n}} R^{\frac{m n-2}{2 m n}+\varepsilon} \sum_{[R]+2 \leq l} \frac{d_{K}^{m}(l)}{l^{1+\varepsilon}} \frac{R}{l-R}\right) \\
= & O_{n, m, \varepsilon}\left(x^{\frac{m n-1}{2 m n}} D_{K}^{\frac{1}{2 n}} R^{-\frac{m n+1}{2 m n}+\varepsilon}+x^{\frac{m n-2}{2 m n}} D_{K}^{\frac{1}{n}} R^{\frac{m n-2}{2 m n}+\varepsilon}\right) .
\end{aligned}
$$

From above results, we obtain

$$
\begin{aligned}
\frac{1}{2 \pi i} \int_{C_{3}} \zeta_{K}(s)^{m} \frac{x^{s}}{s} d s= & \frac{C x^{\frac{m n-1}{2 m n}} D_{K}^{\frac{1}{2 n}}}{2 \pi i} \sum_{l \leq R} \frac{d_{K}^{m}(l)}{l^{\frac{m n+1}{2 m n}}} \tau\left(2 n \pi\left(\frac{l x}{D_{K}^{m}}\right)^{\frac{1}{m n}}\right) \\
& +O_{n, m, \varepsilon}\left(x^{\frac{m n-1}{2 m n}} D_{K}^{\frac{1}{2 n}} R^{-\frac{m n+1}{2 m n}+\varepsilon}+x^{\frac{m n-2}{2 m n}} D_{K}^{\frac{1}{n}} R^{\frac{m n-2}{2 m n}+\varepsilon}\right) .
\end{aligned}
$$

From estimate (2.10) and (2.11), it is obtained that

$$
\begin{aligned}
\Delta_{K}^{m}(x)= & \frac{C x^{\frac{m n-1}{2 m n}} D_{K}^{\frac{1}{2 n}}}{2 \pi i} \sum_{l \leq R} \frac{d_{K}^{m}(l)}{l^{\frac{m n+1}{2 m n}}} \tau\left(2 m n \pi\left(\frac{l x}{D_{K}^{m}}\right)^{\frac{1}{m n}}\right) \\
& +O_{n, m, \varepsilon}\left(x^{\frac{m n-2}{2 m n}+\varepsilon} D_{K}^{\frac{1}{n}+\varepsilon} R^{\frac{m n-2}{2 m n}+\varepsilon}+x^{\frac{m n-1}{m n}+\varepsilon} D_{K}^{\frac{1}{n}+\varepsilon} R^{-\frac{1}{m n}+\varepsilon}\right)
\end{aligned}
$$

Next we consider the above sum. Let $F_{K}=d_{K}^{m} * \mu$, where $*$ is the Dirichlet product symbol. From lemma 2.5 this becomes

$$
\begin{aligned}
& \Delta_{K}^{m}(x)
\end{aligned}
$$

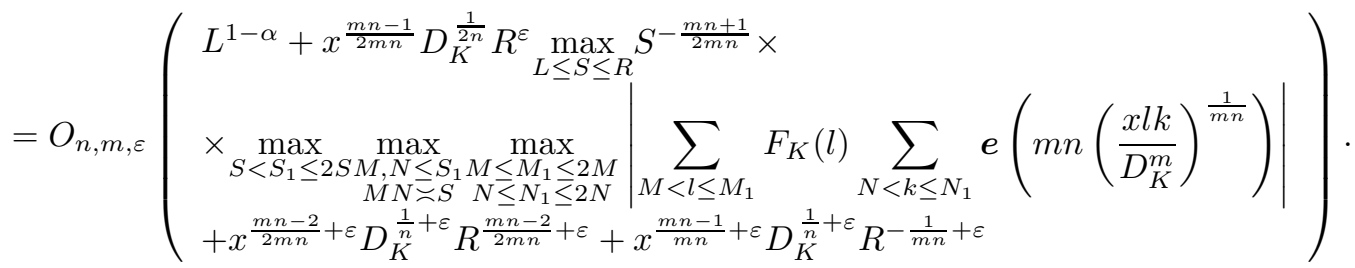


This proves this proposition.

Let $\mathcal{S}_{K}(x, S)$ be the sum in the $O$-term, that is,

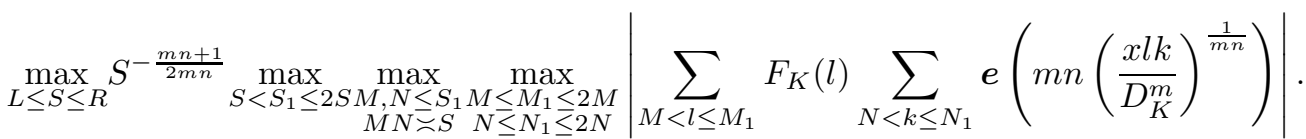

This proposition reduces the initial problem to an exponential sums problem. There are many results to estimate exponential sum. In the next section, we estimate Piltz divisor function by using some results for exponential sum established by many authors.

\section{Estimate OF COUNTING FUNCTION}

In the last section, we show that the error term of Piltz divisor function $\Delta_{K}^{m}(x)$ can be expressed as a exponential sum. Let $X>1$ be a real number, $1 \leq M<$ $M_{1} \leq 2 M$ and $1 \leq N<N_{1} \leq 2 N$ be integers and $\left(a_{m}\right),\left(b_{n}\right) \subset \mathbf{C}$ be sequence of complex numbers, and let $\alpha, \beta \in \mathbf{R}$ and we define

$$
\mathcal{S}=\sum_{M<m \leq M_{1}} a_{m} \sum_{N<n \leq N_{1}} b_{n} \boldsymbol{e}\left(X\left(\frac{m}{M}\right)^{\alpha}\left(\frac{n}{N}\right)^{\beta}\right) .
$$

In $1998 \mathrm{Wu}$ shows this lemma.

Lemma 3.2 (Wu [Wu98). Let $\alpha, \beta \in \mathbf{R}$ such that $\alpha \beta(\alpha-1)(\beta-1) \neq 0$, and $\left|a_{m}\right| \leq 1$ and $\left|b_{n}\right| \leq 1$ and $\mathcal{L}=\log (X M N+2)$. Then

$$
\mathcal{L}^{-2} \mathcal{S}=O\left(\begin{array}{l}
\left(X M^{3} N^{4}\right)^{\frac{1}{5}}+\left(X^{4} M^{10} N^{11}\right)^{\frac{1}{16}}+\left(X M^{7} N^{10}\right)^{\frac{1}{11}} \\
+M N^{\frac{1}{2}}+\left(X^{-1} M^{14} N^{23}\right)^{\frac{1}{22}}+X^{-\frac{1}{2}} M N
\end{array}\right) .
$$

Next Bordellès also shows this lemma by using estimate for triple exponential sums by Robert and Sargos.

Lemma 3.3 (Bordellès Bo15). Let $\alpha, \beta \in \mathbf{R}$ such that $\alpha \beta(\alpha-1)(\beta-1) \neq 0$, and $\left|a_{m}\right| \leq 1$ and $\left|b_{n}\right| \leq 1$. If $X=O(M)$ then

$$
\begin{aligned}
& (M N)^{-\varepsilon} \mathcal{S} \\
= & O\left(\left(X M^{5} N^{7}\right)^{\frac{1}{8}}+N\left(X^{-2} M^{11}\right)^{\frac{1}{12}}+\left(X^{-3} M^{21} N^{23}\right)^{\frac{1}{24}}+M^{\frac{3}{4}} N+X^{-\frac{1}{4}} M N\right) .
\end{aligned}
$$

The following Srinivasan's result is important for our estimating $\Delta_{K}^{m}(x)$.

Lemma 3.4 (Srinivasan [Sr62]). Let $N$ and $P$ be positive integers and $u_{n} \geq 0$, $v_{p}>0, A_{n}$ and $B_{p}$ denote constants for $1 \leq n \leq N$ and $1 \leq p \leq P$. Then there exists $q$ with properties

$$
Q_{1} \leq q \leq Q_{2}
$$

and

$\sum_{n=1}^{N} A_{n} q^{u_{n}}+\sum_{p=1}^{P} B_{p} q^{-v_{p}}=O\left(\sum_{n=1}^{N} \sum_{p=1}^{P} \sqrt[u_{n}+v_{p}]{A_{n}^{v_{p}} B_{p}^{u_{n}}}+\sum_{n=1}^{N} A_{n} Q_{1}^{u_{n}}+\sum_{p=1}^{P} B_{p} Q_{2}^{-v_{p}}\right)$.

The constant involved in $O$-symbol is less than $N+P$.

Srinivasan remarks that the inequality in lemma 3.4 corresponds to the 'best possible' choice of $q$ in the range $Q_{1} \leq q \leq Q_{2}$ Sr62. We apply lemma 3.4 to improve the error term $\Delta_{K}^{m}(x)$. 
Theorem 3.5. For every $\varepsilon>0$ the following estimates hold. When $m n \geq 4$, then

$$
\Delta_{K}^{m}(x)=O_{n, m, \varepsilon}\left(x^{\frac{2 m n-3}{2 m n+1}+\varepsilon} D_{K}^{\frac{2 m}{2 m n+1}+\varepsilon}\right)
$$

as $x$ tends to infinity.

Proof. We note that

$$
\begin{aligned}
& \left|\sum_{M<l \leq M_{1}} F_{K}(l) \sum_{N<k \leq N_{1}} e\left(m n\left(\frac{x l k}{D_{K}^{m}}\right)^{\frac{1}{m n}}\right)\right| \\
= & \left|\sum_{M<l \leq M_{1}} F_{K}(l) \sum_{N<k \leq N_{1}} e\left(m n\left(\frac{x M N}{D_{K}^{m}}\right)^{\frac{1}{m n}}\left(\frac{l}{M}\right)^{\frac{1}{m n}}\left(\frac{k}{N}\right)^{\frac{1}{m n}}\right)\right| .
\end{aligned}
$$

We use the above lemmas with $X=m n\left(\frac{x M N}{D_{K}^{m}}\right)^{\frac{1}{m n}}>0$. Let $0 \leq \alpha \leq \frac{1}{3}$, we consider four cases:

\begin{tabular}{ll}
\hline Case 1. & $S^{\alpha} \ll N \ll S^{\frac{1}{2}}$ \\
Case 2. & $S^{\frac{1}{2}} \ll N \ll S^{1-\alpha}$ \\
Case 3. & $S^{1-\alpha} \ll N$ \\
Case 4. & $N \ll S^{\alpha}$
\end{tabular}

When $S^{\alpha} \ll N \ll S^{\frac{1}{2}}$, we apply lemma 3.2 and this gives

$$
\begin{aligned}
& S^{-\varepsilon} x^{\frac{m n-1}{2 m n}} D_{K}^{\frac{1}{2 n}} \mathcal{S}_{K}(x, S) \\
= & O_{n, m, \varepsilon}\left(\begin{array}{l}
x^{\frac{5 m n-3}{10 m n}} D_{K}^{\frac{3}{10 n}} R^{\frac{2 m n-3}{10 m n}}+x^{\frac{2 m n-1}{4 m n}} D_{K}^{\frac{1}{4 n}} R^{\frac{5 m n-8}{32 m n}} \\
+x^{\frac{11 m n-9}{22 m n}} D_{K}^{\frac{9}{22 n}} R^{\frac{6 m n-9}{22 m n}}+x^{\frac{m n-1}{2 m n}} D_{K}^{\frac{1}{2 n}} R^{\frac{m n-1}{2 m n}-\frac{1}{2} \alpha} \\
+x^{\frac{11 m n-12}{22 m n}} D_{K}^{\frac{6}{11 n}} R^{\frac{15 m n-24}{44 m n}}+x^{\frac{m n-2}{2 m n}} D_{K}^{\frac{1}{n}} R^{\frac{m n-2}{2 m n}}
\end{array}\right) .
\end{aligned}
$$

When $S^{\frac{1}{2}} \ll N \ll S^{1-\alpha}$ we use lemma 3.2 again reversing the role of $M$ and $N$. We obtain the same estimate for the case that $S^{\alpha} \ll N \ll S^{\frac{1}{2}}$.

For the case 3, we use lemma 3.3

$$
\begin{aligned}
& S^{-\varepsilon} x^{\frac{m n-1}{2 m n}} D_{K}^{\frac{1}{2 n}} \mathcal{S}_{K}(x, S) \\
& =O_{n, m, \varepsilon}\left(\begin{array}{l}
x^{\frac{4 m n-3}{8 m n}} D_{K}^{\frac{3}{8 n}} R^{\frac{m n-3}{8 m n}+\frac{1}{4} \alpha}+x^{\frac{3 m n n-4}{6 m n}} D_{K}^{\frac{2}{3 n}} R^{\frac{5 m n-8}{12 m n}+\frac{1}{12} \alpha} \\
+x^{\frac{4 m n-5}{8 m n}} D_{K}^{\frac{5}{8 n}} R^{\frac{3 m n-5}{8 m n}-\frac{1}{12} \alpha} \\
+x^{\frac{m n-1}{2 m n}} D_{K}^{\frac{1}{2 n}} R^{\frac{m n-2}{4 m n}+\frac{1}{4} \alpha}+x^{\frac{2 m n-3}{4 m n}} D_{K}^{\frac{3}{4 n}} R^{\frac{2 m n-3}{4 m n}}
\end{array}\right) .
\end{aligned}
$$

If $x^{\frac{1}{m n(1-\alpha)-1}} D_{K}^{-\frac{m}{m n(1-\alpha)-1}} \ll S$, the condition of Lemma $3.3 X=O(N)$ is satisfied. Therefore it suffices to choose $L=x^{\frac{1}{m n(1-\alpha)-1}} D_{K}^{-\frac{m}{m n(1-\alpha)-1}}$. For the case 4 , we use Lemma 3.3 again reversing the role of $M$ and $N$. We obtain the same estimate for 
WATARU TAKEDA

the case that $N \ll S^{\alpha}$. Combining (3.6) and (3.7) with proposition 2.6, we obtain $(3.8)$

$$
\Delta_{K}^{m}(x)=O_{n, m, \varepsilon}\left(\begin{array}{l}
x^{\frac{5 m n-3}{10 m n}} D_{K}^{\frac{3}{10 n}} R^{\frac{2 m n-3}{10 m n}+\varepsilon}+x^{\frac{2 m n-1}{4 m n}} D_{K}^{\frac{1}{4 n}} R^{\frac{5 m n-8}{32 m n}+\varepsilon} \\
+x^{\frac{11 m n-9}{22 m n}} D_{K}^{\frac{9}{22 n}} R^{\frac{6 m n-9}{22 m n}+\varepsilon}+x^{\frac{m n-1}{2 m n}} D_{K}^{\frac{1}{2 n}} R^{\frac{m n-1}{2 m n}-\frac{1}{2} \alpha+\varepsilon} \\
+x^{\frac{11 m n-12}{22 m n}} D_{K}^{\frac{6}{11 n}} R^{\frac{15 m n-24}{44 m n}+\varepsilon}+x^{\frac{m n-2}{2 m n}} D_{K^{\frac{1}{n}}}^{\frac{m n-2}{2 m n}+\varepsilon} \\
+x^{\frac{4 m n-3}{8 m n}} D_{K}^{\frac{3}{8 n}} R^{\frac{m n-3}{8 m n}+\frac{1}{4} \alpha+\varepsilon}+x^{\frac{3 m n-4}{6 m n}} D_{K}^{\frac{2}{3 n}} R^{\frac{5 m n-8}{12 m n}+\frac{1}{12} \alpha+\varepsilon} \\
+x^{\frac{4 m n-5}{8 m n}} D_{K}^{\frac{5}{8 n}} R^{\frac{3 m n-5}{8 m n}+\frac{1}{12} \alpha+\varepsilon}+x^{\frac{2 m n-3}{4 m n}} D_{K}^{\frac{3}{4 n}} R^{\frac{2 m n n-3}{4 m n}+\varepsilon} \\
+x^{\frac{m n-1}{m n}+\varepsilon} D_{K}^{\frac{1}{n}+\varepsilon} R^{-\frac{1}{m n}+\varepsilon}+x^{\frac{1-\alpha}{m n(1-\alpha)-1}} D_{K}^{-\frac{m}{m n(1-\alpha)}}
\end{array}\right) .
$$

By lemma 3.4 with $x^{\frac{1}{m n(1-\alpha)-1}} D_{K}^{-\frac{m}{m n(1-\alpha)-1}} \leq R \leq x D$ there exists $R$ such that the error term of estimate (3.8) is much less than

$$
\begin{aligned}
& x^{\frac{2 m n}{2 m n+7}+\varepsilon} D_{K}^{\frac{2 m}{2 m n+7}+\varepsilon}+x^{\frac{5 m n+3}{5 m n+24}+\varepsilon} D_{K}^{\frac{5 m}{5 m n+24}+\varepsilon}+x^{\frac{6 m n-4}{6 m n+13}+\varepsilon} D_{K}^{\frac{6 m}{6 m n+13}+\varepsilon} \\
& +x^{\frac{(1-\alpha) m n+\alpha-1}{(1-\alpha) m n+1}+\varepsilon} D_{K}^{\frac{(1-\alpha) m}{(1-\alpha) m n+1}+\varepsilon}+x^{\frac{15 m n-17}{15 m n+20}+\varepsilon} D_{K}^{\frac{3 m}{3 m n+4}+\varepsilon}+x^{\frac{m n-2}{m n}+\varepsilon} D_{K}^{\frac{1}{n}+\varepsilon} \\
& +x^{\frac{(2 \alpha+1) m n-2 \alpha}{(2 \alpha+1) m n+5}+\varepsilon} D_{K}^{\frac{(2 \alpha+1) m}{(2 \alpha+1) m n+5}+\varepsilon}+x^{\frac{(\alpha+5) m n-\alpha-7}{(\alpha+5) m n+4}+\varepsilon} D_{K}^{\frac{(\alpha+5) m}{(\alpha+5) m n+4}+\varepsilon} \\
& +x^{\frac{(2 \alpha+9) m n-2 \alpha-12}{(2 \alpha+9) m n+9}+\varepsilon} D_{K}^{\frac{(2 \alpha+9) m}{(2 \alpha+9) m n+9}+\varepsilon}+x^{\frac{2 m n-3}{2 m n+1}+\varepsilon} D_{K}^{\frac{2 m}{2 m n+1}+\varepsilon} \\
& +x^{\frac{5 m n(1-\alpha)-6+3 \alpha}{10 m n(1-\alpha)-10}+\varepsilon} D_{K}^{\frac{K m-3 m \alpha}{10 m n(1-\alpha)-10}+\varepsilon}+x^{\frac{16 m n(1-\alpha)-19+8 \alpha}{32 m n(1-\alpha)-32}+\varepsilon} D_{K}^{\frac{3 m-8 m \alpha}{32 m n(1-\alpha)-32}+\varepsilon} \\
& +x^{\frac{11 m n(1-\alpha)-14+9 \alpha}{22 m n(1-\alpha)-22}+\varepsilon} D_{K}^{\frac{3 m-9 m \alpha}{22 m n(1-\alpha)-22}+\varepsilon}+x^{\frac{1}{2}+\varepsilon} \\
& +x^{\frac{22 m n(1-\alpha)-31+24 \alpha}{44 m n(1-\alpha)-44}+\varepsilon} D_{K}^{\frac{9 m-24 m \alpha}{44 m(1-\alpha)-44}+\varepsilon}+x^{\frac{m n(1-\alpha)-2+2 \alpha}{2 m n(1-\alpha)-2}+\varepsilon} D_{K}^{\frac{m-2 m \alpha}{2 m n(1-\alpha)-2}+\varepsilon} \\
& +x^{\frac{4 m n(1-\alpha)-6+5 \alpha}{8 m n(1-\alpha)-8}+\varepsilon} D_{K}^{\frac{2 m-5 m \alpha}{8 m n(1-\alpha)-8}+\varepsilon}+x^{\frac{6 m n(1-\alpha)-9+9 \alpha}{12 m n(1-\alpha)-12}+\varepsilon} D_{K}^{\frac{3 m-9 m \alpha}{12 m n(1-\alpha)-12}+\varepsilon} \\
& +x^{\frac{12 m n(1-\alpha)-18+17 \alpha}{24 m n(1-\alpha)-24}+\varepsilon} D_{K}^{\frac{6 m-17 m \alpha}{24 m n(1-\alpha)-24}+\varepsilon}+x^{\frac{2 m n(1-\alpha)-3+3 \alpha}{4 m n(1-\alpha)-4}+\varepsilon} D_{K}^{\frac{m-3 m \alpha}{4 m n(1-\alpha)-4}+\varepsilon} \\
& +x^{\frac{1-\alpha}{m n(1-\alpha)-1}} D_{K}^{-\frac{m(1-\alpha)}{m n(1-\alpha)-1}} \text {. }
\end{aligned}
$$

When $m n \geq 4$ and $\alpha=\frac{m n+3}{7 m n-5}$, then we have

$$
\Delta_{K}^{m}(x)=O_{n, m, \varepsilon}\left(x^{\frac{2 m n-3}{2 m n+1}+\varepsilon} D_{K}^{\frac{2 m}{2 m n+1}+\varepsilon}\right) .
$$

This proves the theorem.

For $m n \geq 4$ this theorem gives new results for Piltz divisor problem over number field. In particular, if we fix $K$ with $[K: \mathbf{Q}]=4$ then we improve the estimate for $\Delta_{K}(x)$ as follows:

Corollary 3.9. For any number field $K$ with $[K: \mathbf{Q}]=4$,

$$
\Delta_{K}(x)=O_{K, \varepsilon}\left(x^{\frac{5}{9}+\varepsilon}\right) .
$$

This result is better than Bordellès' result.

\section{Application}

In this section we introduce some applications of our theorems. First we obtain uniform estimate for ideal counting function $I_{K}(x)$. From the proof of theorem 3.5. we obtain the following theorem. 
Theorem 4.1. For all $\varepsilon>0$ for any fixed $0 \leq \beta \leq \frac{8}{2 n+5}-\varepsilon$ and $C>0$ the followings hold. If $K$ runs through number fields with $[K: \mathbf{Q}] \leq n$ and $D_{K} \leq C x^{\beta}$ then

$$
\Delta_{K}(x)=O_{C, n, \varepsilon}\left(x^{\frac{2 n-3+2 \beta}{2 n+1}+\varepsilon}\right) .
$$

The condition $D_{K} \leq C x^{\beta}$ is caused by the relation between the principal term and the error term. It is well known that $I_{K}(x)$ is very important to estimate the distribution of relatively $r$-prime lattice points. We regard an $\ell$-tuple of ideals $\left(\mathfrak{a}_{1}, \mathfrak{a}_{2}, \ldots, \mathfrak{a}_{\ell}\right)$ of $\mathcal{O}_{K}$ as a lattice point in $K^{\ell}$. We say that a lattice point $\left(\mathfrak{a}_{1}, \mathfrak{a}_{2}, \ldots, \mathfrak{a}_{\ell}\right)$ is relatively $r$-prime for a positive integer $r$, if there exists no prime ideal $\mathfrak{p}$ such that $\mathfrak{a}_{1}, \mathfrak{a}_{2}, \ldots, \mathfrak{a}_{\ell} \subset \mathfrak{p}^{r}$. Let $V_{\ell}^{r}(x, K)$ denote the number of relatively $r$-prime lattice points $\left(\mathfrak{a}_{1}, \mathfrak{a}_{2}, \ldots, \mathfrak{a}_{\ell}\right)$ such that their ideal norm $\mathfrak{N a}_{i} \leq x$.

B. D. Sittinger shows that

$$
V_{\ell}^{r}(x, K) \sim \frac{\rho_{K}^{\ell}}{\zeta_{K}(r \ell)} x^{\ell},
$$

where $\rho_{K}$ is the residue of $\zeta_{K}$ as $s=1$ [Si10. It is well known that

$$
\rho_{K}=\frac{2^{r_{1}}(2 \pi)^{r_{2}} h_{K} R_{K}}{w_{K} \sqrt{D_{K}}}
$$

where $h_{K}$ is the class number of $K, R_{K}$ is the regulator of $K$ and $w_{K}$ is the number of roots of unity in $\mathcal{O}_{K}^{*}$.

After that we show some results for the error term:

$$
E_{\ell}^{r}(x, K)=V_{\ell}^{r}(x, K)-\frac{\rho_{K}^{\ell}}{\zeta_{K}(r \ell)} x^{\ell} .
$$

In [Ta17] and TK17] we consider the relation between relatively $r$-prime problem and other mathematical problems. If we assume the Lindelöf Hypothesis for $\zeta_{K}(s)$, then it holds that for all $\varepsilon>0$

$$
E_{\ell}^{r}(x, K)= \begin{cases}O_{\varepsilon}\left(x^{\frac{1}{r}\left(\frac{3}{2}+\varepsilon\right)}\right) & \text { if } r \ell=2, \\ O_{\varepsilon}\left(x^{\ell-\frac{1}{2}+\varepsilon}\right) & \text { otherwise }\end{cases}
$$

From easy calculation, we obtain the following corollary.

Corollary 4.4. For all $\varepsilon>0$ and for any fixed $0 \leq \beta \leq \frac{8}{2 n+5}-\varepsilon$ and $C>0$ the followings hold. If $K$ runs through number fields with $[K: \mathbf{Q}] \leq n$ and $D_{K} \leq C x^{\beta}$, then

$$
E_{\ell}^{r}(x, K)= \begin{cases}O_{C, n, \varepsilon}\left(x^{\frac{4 n-2}{r(2 n+1)}+\frac{4}{2 n+1} \beta+\varepsilon}\right) & \text { if } r \ell=2, \\ O_{C, n, \varepsilon}\left(x^{\ell-\frac{4}{2 n+1}+\frac{2 n+5-(2 n+1) \ell}{2(2 n+1)} \beta+\varepsilon}\right) & \text { otherwise }\end{cases}
$$

For the proof of this corollary, please see the proof of Theorem 4.1 of [TK17.

\section{Conjecture}

Theorem 4.1 states good uniform upper bounds. It is proposed that for all number fields $K$ the best uniform upper bound of the error term is better than that on the assumption of the Lindelöf Hypothesis (1.6).

Conjecture 5.1. If $K$ runs through number fields with $D_{K}<x$, then

$$
\Delta_{K}^{m}(x)=o\left(x^{\frac{1}{2}}\right)
$$


If $K$ runs through cubic extension fields with $D_{K} \leq C x^{\frac{1}{4}-\varepsilon}$, then this conjecture holds from theorem 4.1

From estimate (1.5), this conjecture may give the best estimate for uniform upper bound of $\Delta_{K}^{m}(x)$. As we remarked above (Theorem 1.2) this conjecture is very difficult even when $K$ is fixed and $m=1$.

\section{REFERENCES}

[At41] F. V. Atkinson. A divisor problem. The Quarterly Journal of Mathematics, 1: 193-200. 1941.

[Bo15] O. Bordellès, On the ideal theorem for number fields, Functiones et Approximatio 53(1): 31-45. 2015.

[BW17] J. Bourgain and N. Watt, Mean square of zeta function, circle problem and divisor problem revisited, Preprint 2017, 23 pp. https://arxiv.org/abs/1709.04340.

[GKMN05] K. Girstmair, M. Kühleitner, W. Müller, and W. G. Nowak, The Piltz divisor problem in number fields: An improved lower bound by Soundararajan's method, Acta Arithmetica, 117: 187-206. 2005.

[Hu00] M. N. Huxley, Integer points, exponential sums and the Riemann zeta function, Number Theory for the Millennium, II: 275-290, 2002.

[La10] H. Lao. On the distribution of integral ideals and Hecke Grössencharacters. Chinese Annals of Mathematics, Series B, 31(3): 385-392. 2010.

[Mü88] W. Müller. On the distribution of ideals in cubic number fields. Monatshefte für Mathematik, 106(3): 211-219. 1988.

[No93] W. G. Nowak. On the distribution of integral ideals in algebraic number theory fields, Math. Nachr. 161: 59-74. 1993.

[Ra59] H. Rademacher, On the Phragmén-Lindelöf theorem and some applications, Math. Z. 72: 192-204. 1959.

[Si10] B. D. Sittinger. The probability that random algebraic integers are relatively r-prime. Journal of Number Theory, 130(1): 164-171. 2010.

[Sr62] B. R. Srinivasan. On Van der Corput's and Nieland's results on the Dirichlet's divisor problem and the circle problem. Proc Natl Inst Sci India, Part A. 28: 732-742. 1962.

[Ta17] W. Takeda. Visible lattice points and the Extended Lindelöf Hypothesis. Journal of Number Theory, 180: 297-309. 2017.

[TK17] W. Takeda and S. Koyama. Estimates of lattice points in the discriminant aspect over abelian extension fields. Forum Mathematicum, 30(3), 767-773. 2018.

[Wu98] J. Wu, On the average number of unitary factors of finite abelian groups, Acta Arithmetica. 84:17-29. 1998.

Department of Mathematics, Nagoya University, Chikusa-ku, Nagoya 464-8602, Japan

E-mail address: d18002r@math.nagoya-u.ac.jp 